\title{
"Safe" oxygen saturation levels in extremely preterm infants: have we found a definite answer?
}

The history of neonatology is filled with fluctuations in relation to treatments used, particularly in preterm infants. From the liberal use of fluids to cover all losses to fluid restriction in order to avoid persistent ductus arteriosus and bronchopulmonary dysplasia (BPD); from the prophylactic closure to living with patent ductus arteriosus without treatment, from prolonged fasting to "reduce" necrotizing enterocolitis to aggressive nutrition to improve the long-term prognosis; these are just a few examples. Changes occurred as the result of bad experiences, in other cases by understanding physiology and, more recently, new approaches respond to the outcome in studies that follow evidence-based medicine guidelines.

Oxygen management in preterm infants is one of the most significant examples of these shifts in the focus.

The initial discovery of oxygen effectiveness to reduce periodic breathing in the 1940s led to its liberal use in preterm infants. ${ }^{1}$ Only after many years, and following a serious epidemics of "retrolental fibroplasia," later known as retinopathy of prematurity (ROP), in 1951 it was deducted that this condition was caused by the excessive use of oxygen. ${ }^{2}$ The response to this situation was a strict restriction on the use of oxygen, which resulted in a dramatic increase of mortality and neurological sequelae. It was estimated that the restrictive use of oxygen led to a ratio of 16 deaths per 1 case of prevented ROP.

More recently, and parallel to the advances in intensive care and the longer survival of preterm infants, an increase in associated morbidities was observed in the most immature infants: pulmonary sequelae (BPD) and a second epidemics of ROP. It was then understood that oxygen concentration had to be adjusted according to the arterial oxygen partial pressure $\left(\mathrm{paO}_{2}\right)$ and / or arterial oxygen saturation. However, what are the ideal oxygen levels for the maintenance of preterm infants to survive with no sequelae?

During the 1990s, oxygen levels used at neonatal units varied worldwide. Differences among sites were significant, even within the same country. In 2001, in England there were sites that accepted minimum saturations of $70 \%$ and maximum saturations of $90 \%$, while others proposed minimum saturations of approximately $90 \%$ and maximum saturations of up to $100 \%$. Patients managed with the latter approach had a higher rate of severe ROP, as well as a more prolonged use of oxygen and mechanical ventilation which was not associated with a better prognosis in terms of survival nor neurological development. ${ }^{3}$

Based on the available information, several randomized studies were developed. The STOP-ROP trial in patients with prethreshold ROP demonstrated that maintaining oxygen saturations at $96-99 \%$ did not improve ROP, as believed, and instead worsened BPD when compared to the group where oxygen saturations were maintained at $89-94 \%{ }^{4}$ In 2003, Askie showed that the attempt to maintain oxygen saturations at $95-98 \%$ versus $91-95 \%$ did not improve neurological development but worsened the lung condition. ${ }^{5}$

These and other studies led to the recommendations made by the American Academy of Pediatrics (AAP) and other associations, which established saturation targets at $85-95 \%$.

But, is it the same for a very immature preterm infant ( $<28$ weeks of gestation) to be exposed to saturations close to $85 \%$ than approaching the $95 \%$ level? In order to determine "safe" oxygen levels within this wide range of extremely preterm infants with $<28$ weeks of gestation, several international, randomized, multicenter trials were conducted simultaneously, such as the SUPPORT trial (NICHD Neonatal Research Network, USA), ${ }^{6}$ the BOOST II trials in the United Kingdom (UK), in Australia (A), and in New Zealand (NZ), ${ }^{7}$ and finally, the Canadian Oxygen Trial (COT), ${ }^{8}$ with the participation of three Argentinean sites, where the authors of this article acted as clinical research coordinators. Researchers agreed on similar protocols for the comparison of children exposed to a saturation of $85-89 \%$ versus $91-94 \%$, and proposed that a highly reliable metaanalysis could be performed at the end of the trial (NeOProM Collaboration). In order to demonstrate significant differences in mortality or severe disability in surviving patients, 5000 preterm infants born at 23-27 weeks of gestation should be included. All clinical trials were blind: to mask the intervention; 
therefore, while the reading of pulse oximeters ("saturometers") was between $88 \%$ and $92 \%$, half of them actually had saturations 3\% higher (91$95 \%)$, and the other half, $3 \%$ lower (85-89\%).

Although the agreed primary outcome measure was survival at 18-24 months (adjusted age) with no major sequelae, some studies also assessed and published short-term results. For us neonatologists and nurses, it was very frustrating to find out in 2010, through the SUPPORT trial, that newborn infants in the group where saturation levels were maintained at $91-94 \%$ had a significantly higher incidence of severe ROP; however, at the same time, patients in the group where saturation was maintained at $85-89 \%$, had a significantly higher mortality, basically related to mortality after the first week. ${ }^{6}$ Based on these results, which are still in the process of being included, safety committees of the BOOST UK and A trials performed interim analyses showing similar effects and arrived at a consensus together with the researchers that the studies had to be terminated early. Recruitment had already been completed in the BOOST NZ trial. The independent safety committee responsible for monitoring the COT trial also performed an interim analysis and recommended to continue with patient enrolment until completing the originally calculated sample size.

In May 2013, the United States Society for Pediatric Research (SPR) submitted and published the results of these studies. The BOOST early findings were similar to those of the SUPPORT trial. ${ }^{6,7}$ The COT demonstrated that, at 18 months of adjusted age, the number of surviving infants with no severe sequelae was similar in both groups. ${ }^{8}$ It is worth mentioning that the results of the assessment of the SUPPORT patients at 1822 months old showed no differences in survival without severe sequelae between groups, as in the COT trial. ${ }^{9}$

A more detailed analysis of the COT trial reflected a trend, which was not statistically significant, towards a late mortality somewhat higher in children exposed to lower saturations. ${ }^{8,10}$

In addition, the detailed readings of all studies show that median saturations to which all infants were finally exposed to in both groups were higher than those set as target saturations. In many sites, this is probably the result of nurses tending to choose values in the upper range so as to reduce the frequency of hypoxemia episodes, which are very common in these patients. Also, during the periods of no oxygen requirement, some preterm infants may have had saturation levels higher than those indicated in the study target saturations. ${ }^{10}$

Additionally, when comparing data, it is very interesting to note that a more adjusted target compliance was achieved in the COT study, with patients staying longer within the target saturation range than those of the SUPPORT trial. The breakdown of median saturation levels between both groups was also higher in the COT study. Lastly, overall mortality was lower in the COT study than in any of the other four studies. ${ }^{6,78}$

So, what should we do? As suggested by Barrington in his web site, and by Polin and Bancalari in their editorial comments, the recommendation is to choose saturation target values of $90-95 \%$, accepting the risk of a higher incidence of severe ROP based on the fact that ROP rarely causes blindness. 10,11 $^{10}$ This may work in sites that are similar to those taking part in the studies, but this is not the case of all sites in Argentina (and probably not in the rest of the countries), where an actual epidemics of blindness caused by ROP has emerged, affecting not only extremely preterm infants. ${ }^{12}$ At present, it would be wise to wait for the BOOST 18-month follow-up results and the final metaanalysis of the five trials. In the meantime, it seems more important to maintain the strict saturation limits proposed by the neonatology and pediatric division for preterm infants exposed to oxygen than knowing what is the accurate figure for determining such levels. It would certainly be logic to avoid saturations lower than $88 \%$ or higher than $94 \%$. Monitor alarms should therefore be set to $89 \%$ and $93 \%$ so that the staff is warned and have enough time to act. Our nurses and physicians need to understand that they should always be around and respond rapidly to the needs of these infants because they are generally highly unstable, require more effort to be devoted and depend on our reaction. Given the trend towards a higher late mortality with lower saturations, it is yet unknown if increasing saturation target levels after the first weeks of life would actually be beneficial. ${ }^{10}$ It is expected that the new technologies to regulate $\mathrm{FiO}_{2}$ in a servocontrolled manner with pulse oximeters, designed by Engineer Nelson Claure and Dr. Eduardo Bancalari, already available for some ventilators, are also fit for other oxygen supply approaches 
(CPAP, hood, nasal cannula) and prove to be effective at maintaining preterm infants within the desired range.

To sum up, a rational use of oxygen is hard to achieve. Evidence-based medicine was not able to solve all problems, but we are certainly on the right track.

Néstor Vain, M.D.,

School of Medicine, Universidad de Buenos Aires. Fundación para la Salud Materno Infantil (Fundasamin). Neonatology and Pediatric Division, Sanatorio de la Trinidad Palermo. nvain@fundasamin.org / nestor.vain@trinidad.com.ar

Cecilia García, M.D.

Neonatal Intensive Care Unit, Sanatorio de la Trinidad Palermo. City of Buenos Aires cecilia.garcia@trinidad.com.ar

http:/ /dx.doi.org/10.5546/aap.2013.372

1. Wilson JL, Long SB, Howard PJ. Respiration of premature infants: response to variations of oxygen and to increased carbon dioxide in inspired air. Am J Dis Child 1942;63:10805.

2. Campbell K. Intensive oxygen therapy as a possible cause of retrolental fibroplasias: a clinical approach. Med J Aust 1951;14;2(2):48-50.
3. Tin W, Milligan DW, Pennefather P, Hey E. Pulse oximetry, severe retinopathy, and outcome at one year in babies of less than 28 weeks gestation. Arch Dis Child Fetal Neonatal Ed 2001;84:F106-F110.

4. Supplemental therapeutic oxygen for prethreshold retinopathy of prematurity (STOP-ROP), a randomized, controlled trial. I: primary outcomes. Pediatrics 2000;105:295-310.

5. Askie LM, Henderson-Smart DJ, Irwig L, Simpson JM. Oxygen-saturation targets and outcomes in extremely preterm infants. N Engl J Med 2003 Sep 4;349(10):959-67.

6. SUPPORT Study Group of the Eunice Kennedy Shriver NICHD Neonatal Research Network. Target ranges of oxygen saturation in extremely preterm infants. $N$ Engl $J$ Med 2010; 362:1959-69.

7. The BOOST II. United Kingdom, Australia, and New Zealand Collaborative Groups. Oxygen saturation and outcomes in preterm infants. $N$ Engl J Med 2013;368(22):2094-104.

8. Schmidt B, Whyte RK, Asztalos E, Moddemann D. et al. for the Canadian Oxygen Trial (COT) Group. Effects of Targeting Higher vs Lower Arterial Oxygen Saturations on Death or Disability in Extremely Preterm Infants: A Randomized Clinical Trial. JAMA 2013;309(20):2111-20.

9. Vaucher YE, Peralta-Carcelen M, Finer NN, Carlo WA et al. Neurodevelopmental outcomes in the early CPAP and pulse oximetry trial. N Engl J Med 2012; 367:2495-504.

10. Bancalari E, Claure N. Oxygenation targets and outcomes in premature infants. JAMA 2013;309(20):2161-2.

11. Polin R, Bateman D. Oxygen-Saturation Targets in Preterm Infants. N Engl J Med 2013;368(22):2141-2.

12. Lomuto C, Galina L, Brussa M, Quiroga A et al. Epidemiología de la retinopatía del prematuro en servicios públicos de la Argentina durante 2008. Arch Argent Pediatr 2010;108(1):24-30.

\section{Adaptive designs in clinical research}

In research, it is essential to carefully plan all the steps to be taken. Likewise, it is important to consider and define, in advance and in full, all the key elements of the trial. Such planning is based on facts assumed as real or certain, therefore a considerable part of the success of the trial lies on the accuracy of this initial assumption.

Unfortunately, not everything goes as originally planned. Many times, some of the considered endpoints do not behave as expected and, regardless of everything else working out smoothly, the study fails or is seriously compromised.

In an attempt to limit the impact of these circumstances on trials, the so called "adaptive designs" became popular in the past recent years. However, it is worth clarifying that such designs were initially envisaged as a variation to shorten the trial period in the development of a new drug. ${ }^{1}$
What are adaptive designs? Also known as "flexible designs," they are defined as studies that include a prospectively planned opportunity for modification of one or more specified aspects of the trial design based on the analysis of data from subjects in the same study (interim analysis). ${ }^{2}$ In other words, it should be established that if a certain issue occurs in a trial, instead of conducting procedure " $\mathrm{A}$," as initially planned, " $\mathrm{B}$ " should be the approach used. It is relevant to note that any change to be introduced will adjust to clearly pre-specified rules. Although such designs offer certain flexibility, it is a limited flexibility because any change must have been considered in the initial project.

What are adaptive designs for? This type of design allows to modify the original plan and thus avoid failure of the study to meet its goals. This includes the possibility of modifying the sample size, study duration, treatment group 
allocation, number of treatment arms, or study endpoints. ${ }^{3}$

When are adaptive designs used? Adaptive designs are most commonly used in the initial phases of drug development in exploratory studies (for example, studies evaluating toxic doses). In more advanced phases of pharmacological research, adaptive designs can be used in combined phase studies, for adaptive randomization, group sequential designs, sample size re-estimation, or a combination of these. ${ }^{4}$ (Figure 1)

Combined phase studies have become very popular. It is very common to combine Phases I and II so that the study includes the initial drug research in a small group of human beings and the subsequent safety study in a larger population, if safety interim data obtained in the first phase thus allows it.

This can be a covariate adaptive randomization (where the likelihood of being allocated to an arm varies in order to minimize the impact of an asymmetrical distribution of potential confounding factors or an unequal "co-variation") or an outcome adaptive randomization (where the likelihood of being allocated to an arm increases if the preceding subject response was favorable). In a group sequential design the decision to continue or to stop a trial is based on the results obtained in the previous arm. Sample size re-estimation is used when actual significant frequencies are verified as different from those used to estimate the initial sample size, and it should be especially considered when demographic distribution data are not well known.

What are the limitations of adaptive designs? In spite of their increasing popularity, adaptive designs are not welcome by all. It is worth noting that seeking a higher flexibility than that established by the protocol is a risk that also jeopardizes research reliability. The concern is that, after applying several adaptations, the population included in the analysis will be remarkably different from that originally targeted and, as a result, there will be an inadequate control of type I error (to mistakenly attribute effectiveness to an actually ineffective treatment). ${ }^{5}$ This is a source of concern even for the United States national regulatory agency, the Food and Drug Administration. ${ }^{2}$ In addition, planning an adaptive design is a hurdle and poses its own statistical, operational, logistic, and regulatory issues. ${ }^{6}$

Figure 1. Summary of different types of adaptive designs for clinical trials. (From Kairalla JA, et al., 20124. Reproduced under a Creative Commons Attribution License)

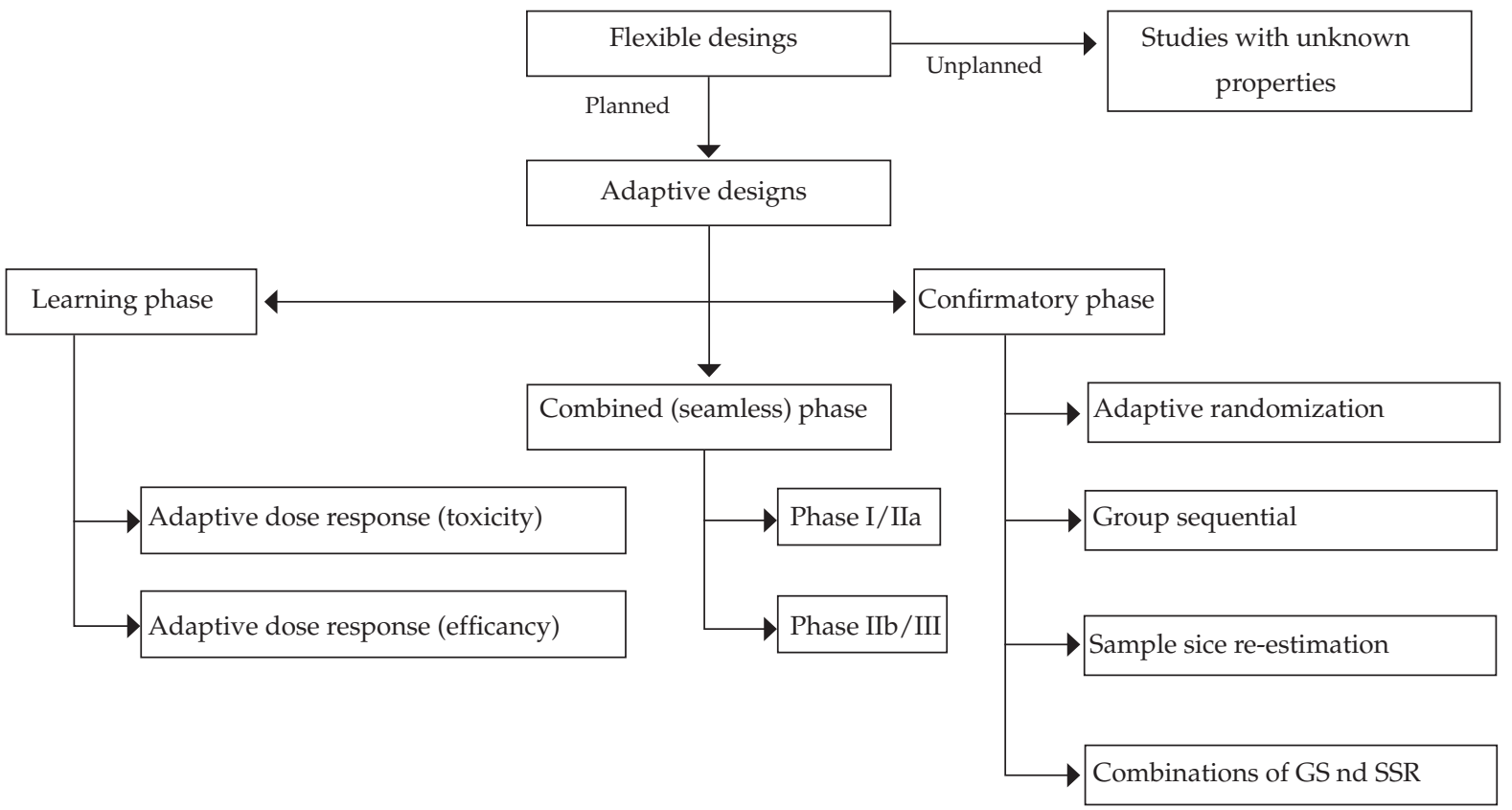


However, when adaptive designs are judiciously used and within their particular scopes, they can certainly be an alternative to be considered in very specific circumstances.

María Fabiana Ossorio, MD Fernando Ferrero, MD

Research and Teaching Hospital General de Niños Pedro de Elizalde, Buenos Aires, Argentina

http:/ /dx.doi.org/10.5546/aap.2013.374

\section{REFERENCES}

1 Schmidt C. Adaptive design may hasten clinical trials. J Natl Cancer Inst 2007; 99(2):108-9.

2. U.S. Food and Drug Administration: Draft Guidance for Industry: adaptive design clinical trials for drugs and biologics. Accessed on 05/07/2012. Available at: http:/ / www. fda.gov/downloads/DrugsGuidanceComplianceRegulatoryInformation/Guidances/UCM201790.pdf.

3. Chow SC, Corey R. Benefits, challenges and obstacles of adaptive clinical trial designs. Orphanet J Rare Dis 2011; 6:79.

4. Kairalla JA, Coffey CS, Thomann MA, Muller KE. Adaptive trial designs: a review of barriers and opportunities. Trials 2012; 13:145.

5. Chow SC, Chang M. Adaptive design methods in clinical trials - a review. Orphanet J Rare Dis 2008; 3:11.

6. Brahmachari B, Bhatt A. Adaptive design - an innovative tool in drug development. Indian J Med Res 2011; 133:243-5. 